Pacific Journal of Mathematics

THE ESSENTIAL SPECTRUM OF A CLASS OF ORDINARY 


\title{
THE ESSENTIAL SPECTRUM OF A CLASS OF ORDINARY DIFFERENTIAL OPERATORS
}

\author{
E. BALsLev AND T. W. GAmelin
}

Introduction. The purpose of this paper is to give a method of determining the essential spectrum of a class of ordinary differential operators in $L^{p}$ of an interval with $\infty$ as a singular endpoint. The method relies on the mapping theorem for the essential spectrum, proved for ordinary differential operators by Rota [9]. A discussion of this type of theorem is presented in $\S 1$. The essential spectrum of the constant coefficient operator and the Euler operator is determined in $\S 4$. It is found that the essential spectrum of the Euler operator is an algebraic curve which varies with the index $p, 1<p<\infty$.

In $\$ \S 5$ and 6 the class of differential operators which are compact with respect to the constant coefficient operator, or Euler operator, is determined. By a fundamental theorem of perturbation theory, these operators may be added to the original operator without altering the essential spectrum.

The results apply to differential equations of Fuchsian type. This includes the Riemann differential equation, whose spectral theory was investigated by Rota [10].

1. Spectral mapping theorems. Let $A$ be a closed, denselydefined operator in a Banach space $\mathfrak{X} . A$ is a Fredholm operator if the null space $\mathscr{N}(A)$ of $A$ is finite dimensional and the range $\mathscr{R}(A)$ of $A$ is closed and of finite codimension in $\mathfrak{X}$. The Fredholm index of $A$ is the number

$$
\kappa(A)=\operatorname{dim} \mathscr{N}(A)-\operatorname{codim} \mathscr{R}(A) .
$$

A complex number $\lambda$ is in the essential resolvent set of $A$, denoted by $\rho_{e}(A)$, if $\lambda I-A$ is a Fredholm operator. Otherwise $\lambda$ is in the essential spectrum of $A$, denoted by $\sigma_{e}(A), \rho(A)$ and $\sigma(A)$ will denote the resolvent set and spectrum of $A$ respectively.

Let $B(\mathfrak{X})$ denote the ring of bounded operators on $\mathfrak{X}$, and let $\mathscr{C}$ denote the ideal of compact operators in $\mathscr{B}(\mathfrak{X}) . \mathscr{A}=\mathscr{B}(\mathfrak{X}) / \mathscr{C}$ is a Banach algebra. The coset $A+\mathscr{C}$ of an element $A \in \mathscr{B}(\mathfrak{X})$ will be denoted by $\widetilde{A}$, and its spectrum will be denoted by $\operatorname{sp}(\widetilde{A})$. The invertible elements of $\mathscr{A}$ are the cosets $\widetilde{B}=B+\mathscr{C}$, where $B \in \mathscr{B}(\mathfrak{X})$ is a Fredholm operator (cf [1]). In particular, $s p(\widetilde{A})=\sigma_{e}(A)$ for all $A \in \mathscr{B}(\mathfrak{X})$. 
Lemma 1. Let $A \in \mathscr{B}(\mathfrak{X})$, and let $f$ be analytic in a neighborhood of $\sigma(A)$. Then $\sigma_{e}(f(A))=f\left(\sigma_{e}(A)\right)$. If $\mu \in \rho_{e}(A)$, then

$$
\kappa(\mu I-f(A))=\Sigma\left\{\kappa(\lambda I-A): \lambda \in f^{-1}(\mu)\right\},
$$

where $\lambda$ is counted in the set $f^{-1}(\mu)$ according to its multiplicity as a solution of $f(z)-\mu=0$.

Proof. The first assertion of the lemma is a trivial consequence of the spectral mapping theorem for Banach algebras:

$$
\sigma_{e}(f(A))=s p\left(\widetilde{f(\widetilde{A}))}=\operatorname{sp}(f(\widetilde{A}))=f(\operatorname{sp}(\widetilde{A}))=f\left(\sigma_{e}(A)\right) .\right.
$$

By replacing $f$ by $\mu-f$ it suffices to establish the formula

$$
\kappa(f(A))=\Sigma\left\{\kappa(\lambda I-A): \lambda \in f^{-1}(0)\right\} .
$$

We can decompose the spectrum of $A$ into a finite number of spectral (closed and open) subsets $F_{i}, i=1, \cdots, n$, such that $f$ is analytic in an open connected neighborhood of each $F_{i}$. Corresponding to each spectral set $F_{i}$, there is a projection $E_{i}$ onto a closed invariant subspace $\mathfrak{X}_{i}$ of $\mathfrak{X}$ such that $I=\sum_{i=1}^{n} E_{i}, E_{i} E_{j}=0, i \neq j$, and $\sigma\left(A \mid \mathfrak{X}_{i}\right)=$ $F_{i}$ (cf [5], VII. 3).

Since the index $\kappa$ satisfies the appropriate additivity conditions, it suffices to prove the formula for the restriction operators $A \mid \mathfrak{X}_{i}$, i.e. we may assume that $f$ is analytic in a connected open neighborhood of $\sigma(A)$.

If $f$ is identically zero, then $f(A)=0$ is Fredholm, so $\mathfrak{X}$ is finite dimensional, and the result is trivial. If $f$ is not identically zero, it has a finite number of zeros $z_{1}, \cdots, z_{n} \in \sigma(A)$, counted according to their multiplicity. Let

$$
g(z)=f(z) /\left(z_{1}-z\right) \cdots\left(z_{n}-z\right) .
$$

$g$ is analytic and nonzero in a neighborhood of $\sigma(A)$, so $g(A)$ is invertible and has index zero. Now

$$
f(A)=\left(z_{1} I-A\right) \cdots\left(z_{n} I-A\right) g(A),
$$

where the $z_{i} I-A$ are Fredholm. Since the index of a product of Fredholm operators is the sum of their indices, we have

$$
\begin{aligned}
\kappa(f(A)) & =\sum_{i=1}^{n} \kappa\left(z_{i} I-A\right) \\
& =\Sigma\left\{\kappa(\lambda I-A): \lambda \in f^{-1}(0) \cap \sigma(A)\right\} \\
& =\Sigma\left\{\kappa(\lambda I-A): \lambda \in f^{-1}(0)\right\} .
\end{aligned}
$$

If $A$ and $B$ are unbounded operators with domains $\mathscr{D}(A)$ and 
$\mathscr{D}(B)$, then their product is defined by

$$
\mathscr{D}(A B)=\{x \in \mathscr{D}(B): B x \in \mathscr{D}(A)\},(A B) x=A(B x) .
$$

$A$ and $B$ commute if $A B=B A$.

If $A$ and $B$ are closed, densely-defined Fredholm operators, then $A B$ is closed and densely-defined, $A B$ is Fredholm, and $\kappa(A B)=\kappa(A)+$ $\kappa(B)$ (cf [6]). Conversely, if $\left\{A_{i}\right\}_{i=1}^{n}$ is a commuting set of closed operators such that $A=A_{1} \cdots A_{n}$ is closed, densely-defined and Fredholm, then each of the $A_{i}$ is densely-defined and Fredholm. For $\mathscr{N}(A) \supseteqq \mathscr{N}\left(A_{i}\right)$ and $\mathscr{R}(A) \subseteq \mathscr{R}\left(A_{i}\right)$ for each $i$. As a special case of these remarks, we can state a version of Lemma 1 for unbounded operators. For ordinary differential operators, the spectral mapping theorem is due to Rota [9].

Lemma 2. Let $A$ be a closed, densely-defined operator in $\mathfrak{X}$, and let $p$ be a polynomial of degree $n$.

(a) If $p\left(\sigma_{e}(A)\right)$ is not the entire complex plane, then $p(A)$ is densely defined and closed.

(b) If $p(A)$ is densely defined and closed, then $\sigma_{e}(p(A))=p\left(\sigma_{e}(A)\right)$. If $\mu \in \rho_{e}(p(A))$,

$$
\kappa(\mu I-p(A))=\sum_{i=1}^{n} \kappa\left(\lambda_{i} I-A\right),
$$

where $\lambda_{1}, \cdots, \lambda_{n}$ are the solutions of $p(z)-\mu=0$, counted according to their multiplicity.

Proof. $\quad \mu I-p(A)=\left(\lambda_{1} I-A\right) \cdots\left(\lambda_{n} I-A\right)$, where the $\lambda_{i} I-A$ commute. If $\mu \notin p\left(\sigma_{e}(A)\right)$, then each $\lambda_{i}$ is in $\rho_{e}(A)$, so $\mu I-p(A)$ is densely-defined and closed. Hence $p(A)$ is densely-defined and closed.

Part (b) of the lemma is a consequence of the preceeding discussion.

2. Some basic facts about linear operators. Let $A$ be a closed densely-defined linear operator in a Banach space $\mathfrak{X}$. The domain $\mathscr{D}(A)$ of $A$ becomes a Banach space when endowed with the $A$-topology, or graph topology, defined by the norm $\|x\|_{A}=\|x\|+\|A x\|$. A linear operator $B: \mathscr{D}(B) \rightarrow \mathfrak{X}$ is said to be A-defined if $\mathscr{D}(B) \supseteqq \mathscr{D}(A) . \quad B$ is $A$-bounded if the restriction of $B$ to $\mathscr{D}(A)$ is a bounded operator from $\mathscr{D}(A)$, with the graph topology, to $\mathfrak{X}$. Its $A$-norm $\|B\|_{A}$ is given by

$$
\|B\|_{A}=\sup _{x \in \mathscr{D}(A)}\left\{\|B x\| /\|x\|_{A}\right\}
$$

$B$ is $A$-compact if it is compact as an operator from $\mathscr{D}(A)$, with the graph topology, to $\mathfrak{X}$. 
If $A^{\prime}$ is a second operator which is closed on $\mathscr{D}(A)=\mathscr{D}\left(A^{\prime}\right)$, then the $A^{\prime}$-topology for $\mathscr{D}(A)$ coincides with the $A$-topology for $\mathscr{D}(A)$. The following lemma gives criteria for $A^{\prime}=A+B$ to be closed on $\mathscr{D}(A)$, and collects certain facts which will be used later.

Lemma 3. Let $A$ be a closed densely-defined operator in $\mathfrak{X}$, and let $B$ be an $A$-defined (not cecessarily closed) linear operator in $\mathfrak{X}$.

(a) If there exist $0 \leqq \alpha<1$ and $0 \leqq \beta$ such that

$$
\|B x\| \leqq \alpha\|A x\|+\beta\|x\| \text { for } x \in \mathscr{D}(A),
$$

then $A+B$ is closed on $\mathscr{D}(A)$.

(b) If $B$ is A-compact, then $A+B$ is closed on $\mathscr{D}(A)$, and $\sigma_{e}(A+B)=\sigma_{e}(A)$,

$$
\kappa(A+B-\lambda I)=\kappa(A-\lambda I) \text { for } \lambda \in \rho_{e}(A) .
$$

(c) If $\lambda \in \rho_{e}(A)$, then there is an $\varepsilon(\lambda)>0$ such that $\|B\|_{A}<\varepsilon(\lambda)$ implies $\lambda \in \rho_{e}(A+B)$.

(d) If $B$ is closed and A-compact, then for every $\varepsilon>0$, there is $a K(\varepsilon)>0$ such that

$$
\|B x\| \leqq \varepsilon\|A x\|+K(\varepsilon)\|x\|, \quad x \in \mathscr{D}(A) .
$$

Proof. (a), (b) and (c) are well-known. Suppose that (d) is not. true. Then there is an $\varepsilon>0$ and a sequence $\left\{x_{n}\right\}$ in $\mathscr{D}(A)$ such that.

$$
\left\|B x_{n}\right\| \geqq \varepsilon\left\|A x_{n}\right\|+n\left\|x_{n}\right\| \text {. }
$$

Since the inequality is homogeneous, we may assume $\left\|x_{n}\right\|_{A}=1$. Passing to a subsequence, if necessary, we may assume, that $B x_{n}$ converges to $y$. Since

$$
\left\|B x_{n}\right\| \geqq \varepsilon\left\|x_{n}\right\|_{A}+(n-\varepsilon)\left\|x_{n}\right\|=\varepsilon+(n-\varepsilon)\left\|x_{n}\right\|,
$$

$x_{n}$ converges to 0 . Since $B$ is closed, $y=0$. On the other hand, $\|y\|=\lim \left\|B x_{n}\right\| \geqq \varepsilon$, a contradiction.

The argument establishing part (d) can be found in [4], p. 39. There are operators $B$ which are $A$-compact but for which no inequality of the form $\|B x\| \leqq \varepsilon\|A x\|+K(\varepsilon)\|x\|$ obtains.

3. Differential operators. Let $(\alpha, \beta)$ be an interval, where $\alpha=-\infty$ and $\beta=+\infty$ are allowed as endpoints. A formal differential, expression $l$ on the interval $(\alpha, \beta)$ is an expression of the form

$$
(l f)(t)=\sum_{j=0}^{n} a_{j}(t) f^{(j)}(t),
$$

where the $a_{j}$ are complex-valued measurable functions on $(\alpha, \beta)$. 
The maximal operator $L$ in $L^{p}(\alpha, \beta), 1<p<\infty$, associated with $l$, is defined by

$\mathscr{D}(L)=\left\{f \in L^{p}(\alpha, \beta): f^{(j)}\right.$ exist and are loc. a.c., $0 \leqq j \leqq n-1$, $\left.l(f) \in L^{p}(\alpha, \beta)\right\}$ and

$$
L f=l(f), \quad f \in \mathscr{D}(L) .
$$

The operator $L_{0}^{\prime}$ is the restriction of $L$ to $C^{\infty}$ functions with compact support contained in $(\alpha, \beta)$.

If $L_{0}^{\prime}$ is closable, then the minimal operator $L_{0}$ associated with $l$ is the closure of $L_{0}^{\prime}$. A differential operator associated with $l$ is an operator $L_{u}$ such that

$$
\mathscr{D}\left(L_{0}\right) \subseteq \mathscr{D}\left(L_{u}\right) \subseteq \mathscr{D}(L)
$$

and

$$
L_{u} f=l(f), \quad f \in \mathscr{D}\left(L_{u}\right) .
$$

Under mild restrictions on the coefficients $a_{j}(t)$, for instance, that $a_{j}(t)$ be locally integrable, $0 \leqq j \leqq n-1$, and that $1 / a_{n}(t)$ be locally integrable, the maximal operator $L$ is densely defined and closed. In this case, $\mathscr{D}\left(L_{0}\right)$ is of finite codimension in $\mathscr{D}(L)$.

Any finite dimensional extension of a Fredholm operator is again Fredholm (cf [6]). Hence, under the preceeding restrictions on the coefficients $a_{j}, \rho_{e}\left(L_{u}\right)=\rho_{e}(L)$ for all differential operators $L_{u}$ determined by $l$. This set is called the essential resolvent set of $l$, and denoted by $\rho_{e}(l)$. Its complement $\sigma_{e}(l)$ is the essential spectrum of $l$.

If $\mathscr{D}\left(L_{u}\right)$ is of codimension $k$ in $\mathscr{D}(L)$, and $\mu \in \rho_{e}(l)$, then $\kappa\left(\mu I-L_{u}\right)=\kappa(\mu I-L)-k$ (cf [6]). To determine the Fredholm index of $\mu I-L_{u}$, it suffices then to find the index of $\mu I-L$, or of $\mu I-L_{0}$.

In the following, $D_{0}$ and $D$ will denote respectively the minimal and maximal operators in $L^{p}(\alpha, \beta)$ determined by the differential expression $(l f)(t)=f^{\prime}(t)$, where $(\alpha, \beta)$ is the interval under consideration.

4. The basic formulae for the essential spectrum.

THEOREM 1. Let $M$ be the maximal differential operator in $L^{p}[0, \infty)$ associated with the expression

$$
(m f)(t)=\sum_{j=0}^{n} a_{j} f^{(j)}(t)
$$

$a_{j}$ constants. 
Let $\pi$ be the polynomial

$$
\pi(z)=\sum_{j=0}^{n} a_{j} z^{j}
$$

Then

$$
\sigma_{e}(m)=\{\pi(i r):-\infty<r<\infty\} .
$$

If $\lambda \in \rho_{e}(m)$, the Fredholm index $\kappa(\lambda I-M)$ is the number of roots of $\pi(z)=\lambda$, counted according to their multiplicity, which lie in the half-plane $\mathscr{R e}(z)<0$.

Proof. The equation $\lambda g-D_{0} g=f$ is satisfied by

$$
g(s)=\left(\lambda I-D_{0}\right)^{-1} f(s)=-e^{\lambda s} \int_{0}^{s} e^{-\lambda t} f(t) d t .
$$

If $\mathscr{R e}(\lambda)<0$, then $\left(\lambda I-D_{0}\right)^{-1} f=k * f$, where $k \in L^{1}(-\infty, \infty)$. So $\left(\lambda I-D_{0}\right)^{-1}$ is bounded, and $\lambda \in \rho\left(D_{0}\right)$. In particular, $\kappa\left(\lambda I-D_{0}\right)=0$ for $\mathscr{R}_{e}(\lambda)<0$.

If $\mathscr{R e}(\lambda)>0$, the adjoint differential equation of $\lambda f=D_{0} f$ has the solution $e^{-\lambda t} \in L^{q}[0, \infty)$, which must be orthogonal to the range of $\lambda I-D_{0}$. If $f \in \mathscr{R}\left(\lambda I-D_{0}\right)$,

$$
\left(\lambda I-D_{0}\right)^{-1} f(s)=e^{\lambda s} \int_{s}^{\infty} e^{-\lambda t} f(t) d t .
$$

This is again a convolution operator with an $L^{1}$-kernel, and so $\left(\lambda I-D_{0}\right)^{-1}$ is bounded on $\mathscr{R}\left(\lambda I-D_{0}\right)$. It follows that $\mathscr{R}\left(\lambda I-D_{0}\right)$ is the subspace of $L^{p}[0, \infty)$ orthogonal to $e^{-\lambda t}$, and so is closed and of codimension 1 in $L^{p}[0, \infty)$. Hence $\lambda \in \rho_{e}\left(D_{0}\right)$ and $\kappa\left(\lambda I-D_{0}\right)=-1$ for $\mathscr{R e}(\lambda)>0$.

Since the Fredholm index is constant on each component of $\rho_{e}\left(D_{0}\right)$, the line $\mathscr{R} e(\lambda)=0$ must be the essential spectrum of $D_{0}$. Since $D$ is an extension of $D_{0}$ by one dimension, $\kappa(\lambda I-D)=1$ if $\mathscr{R e}(\lambda)<0$ and $\kappa(\lambda I-D)=0$ if $\mathscr{R e}(\lambda)>0$.

This establishes the theorem for the special case of the operator $D$. It suffices now to prove that $M=\pi(D) ;{ }^{1}$ then the general result follows from Lemma 2. From the inequality of Lemma 5 we derive the inequality

$$
\left\|D^{n} f\right\| \leqq K\{\|M f\|+\|f\|\}, \quad f \in C_{0}^{\infty}(0, \infty) .
$$

Thus, the $M$-norm and $D^{n}$-norm on $C_{0}^{\infty}(0, \infty)$ are equivalent, and it follows, that

$$
\mathscr{D}\left(M_{0}\right)=\mathscr{D}\left(D_{0}^{n}\right)=\mathscr{D}\left(\pi\left(D_{0}\right)\right) .
$$

Since $M$ is an extension of $\pi(D)$, and since $\operatorname{dim} \mathscr{D}(\pi(D)) / \mathscr{D}\left(\pi\left(D_{0}\right)\right) \geqq n$. it suffices to show, that $\operatorname{dim} \mathscr{D}(M) / \mathscr{D}\left(M_{0}\right)=n$.

\footnotetext{
1 Professor S. Goldberg pointed out, that a proof was missing here.
} 
Since $\mathscr{D}(M)=\mathscr{D}(M-\lambda I)$, we may assume, by altering the constant term of $\pi$, that $M$ is Fredholm. Then

$$
\begin{aligned}
\operatorname{dim} \mathscr{D}(M) / \mathscr{D}\left(M_{0}\right) & =\operatorname{dim} \mathscr{N}(M)+\operatorname{codim} \mathscr{R}\left(M_{0}\right) \\
& =\operatorname{dim} \mathscr{N}(M)+\operatorname{dim} \mathscr{N}(L)
\end{aligned}
$$

where $L$ is the maximal differential operator associated with the adjoint expression (cf. [9]).

We may also assume, that the roots $\lambda_{1}, \cdots, \lambda_{n}$ of $\pi(z)=0$ have distinct real parts. Then $\mathscr{N}(M)$ is spanned by the exponentials $e^{\lambda_{i} t}$, and $\mathscr{N}(L)$ is spanned by the exponentials $e^{-\lambda_{i} t}$. From this it is easy to conclude that $\operatorname{dim} \mathscr{N}(M)+\operatorname{dim} \mathscr{N}(L)=n$.

THEOREM 2. Let the Euler differential expression $l$ on the interval $[1, \infty)$ be defined by

$$
(l f)(t)=\sum_{k=0}^{n} b_{k} t^{k} f^{(k)}(t),
$$

where the $b_{k}$ are constants. Let $L$ be the associated maximal operator in $L^{p}[1, \infty), 1<p<\infty$. Let $d$ be the polynomial

$$
d(z)=b_{0}+\sum_{k=1}^{n} b_{k} \prod_{j=0}^{k-1}\left(z-\left(\frac{1}{p}+j\right)\right) .
$$

Then $\sigma_{e}(l)=\{d(i r):-\infty<r<\infty\}$. For $\lambda \in \rho_{e}(l)$, the Fredholm index $\kappa(\lambda I-L)$ is the number of roots of $d(z)-\lambda=0$, counted according to their multiplicity, which lie in the half-plane $\mathscr{R}_{e}(z)<0$.

Proof. For $f \in L^{p}[1, \infty)$, we define

$$
(\pi f)(s)=e^{s / p} f\left(e^{s}\right), 0 \leqq s<\infty .
$$

It is easily verified, that $\tau$ is an isometric isomorphism of $L^{p}[1, \infty$ ) and $L^{p}[0, \infty)$. Its inverse is given by

$$
f(t)=\left(\tau^{-1} g\right)(t)=t^{-1 / p} g(\log t), 1 \leqq t<\infty .
$$

We have

$$
\begin{aligned}
\frac{d f}{d t} & =t^{-(1 / p)-1} g^{\prime}(\log t)-\frac{1}{p} t^{-(1 / p)-1} g(\log t) \\
& =t^{-(1 / p)-1}\left[\left(\frac{d}{d s}-\frac{1}{p}\right) g(s)\right]_{s=\log t} .
\end{aligned}
$$

By induction on $k$, the following formula obtains

$$
\begin{gathered}
\frac{d^{k} f}{d t^{k}}=t^{-(1 / p)-1}\left[\left(\frac{d}{d s}-\left(\frac{1}{p}+k-1\right)\right)\left(\frac{d}{d s}-\left(\frac{1}{p}+k-2\right)\right) \cdots\right. \\
\left.\times\left(\frac{d}{d s}-\frac{1}{p}\right) g(s)\right]_{s=\log t} .
\end{gathered}
$$


Therefore

$$
\left(\tau t^{k} \frac{d^{k} f}{d t^{k}}\right)(s)=\prod_{j=0}^{k-1}\left(\frac{d}{d s}-\left(\frac{1}{p}+j\right)\right) \tau f(s) .
$$

Let $l_{k}$ be the differential expression

$$
\left(l_{k} f\right)(t)=t^{k} f^{(k)}(t), 1 \leqq t<\infty,
$$

and let $L_{k}$ be the corresponding maximal operator in $L^{p}[1, \infty)$. Then

$$
L_{k}=\tau^{-1} \prod_{j=0}^{k-1}\left(D-\left(\frac{1}{p}+j\right)\right) \tau, k \geqq 1 .
$$

Consequently,

$$
L=\tau^{-1}\left[b_{0} I+\sum_{k=1}^{n} b_{k} \prod_{j=0}^{k-1}\left(D-\left(\frac{1}{p}+j\right)\right)\right] \tau .
$$

Since the essential spectrum and Fredholm index remain invariant under isometric isomorphisms, the result follows from Theorem 1.

REMARK. The essential spectrum of $L$ could also be computed by writing $L$ as a polynomial in the operator $x(d / d x)$, which has the essential spectrum $\{-(1 / p)+i r,-\infty<r<\infty\}$. The Euler operator was originally represented as a polynomial by George Boole.

5. Perturbation of the constant coefficient operator. The inequalities, on which the results of this section are based, are essentially special cases of similar estimates for elliptic partial defferential operators (cf [4]). Similar results for perturbation of partial differential operators are obtained in [3]. For $p=2$ theorems of this type for elliptic operators, including Lemma 7, are proved by Birman (cf. [11]).

Lemma 4. Given $\varepsilon>0$, there exists a constant $K$, depending only on $p$ and $\varepsilon$, such that

$$
\int_{N}^{\infty}|b(t) f(t)|^{p} d t \leqq\left\{\sup _{N \leqq s<\infty} \int_{s}^{s+1}|b(t)|^{p} d t\right\}\left\{\varepsilon \int_{N}^{\infty}\left|f^{\prime}(t)\right|^{p} d t+K \int_{N}^{\infty}|f(t)|^{p} d t\right\}
$$

for all $N \geqq 0$, all functions $b$ locally in $L^{p}[0, \infty)$, and all functions $f$ in the domain of the maximal operator $D$ in $L^{p}[0, \infty)$.

Proof. Let $r$ be a small positive number Let $a$ be a continuously differentiable function on $[0, r]$ such that

$$
0 \leqq a \leqq 1, a(0)=1 \text { and } a(r)=0 .
$$

If $f \in \mathscr{D}(D)$, then 


$$
\begin{gathered}
f(t)=-\int_{0}^{r} \frac{d}{d s}(a(s) f(t+s)) d s \\
=-\int_{0}^{r} a(s) f^{\prime}(t+s) d s-\int_{0}^{r} a^{\prime}(s) f(t+s) d s ; \\
|f(t)| \leqq \int_{0}^{r}\left|f^{\prime}(t+s)\right| d s+K_{0} \int_{0}^{r}|f(t+s)| d s \\
\leqq r^{1 / q}\left\{\int_{0}^{r}\left|f^{\prime}(t+s)\right|^{p} d s\right\}^{1 / p}+K_{0} r^{1 / q}\left\{\int_{0}^{r}|f(t+s)|^{p} d s\right\}^{1 / p} \\
\leqq c_{p} r^{1 / q}\left\{\int_{0}^{r}\left|f^{\prime}(t+s)\right|^{p} d s+K_{0} \int_{0}^{r}|f(t+s)|^{p} d s\right\}^{1 / p},
\end{gathered}
$$

where $(1 / p)+(1 / q)=1$.

If $r$ is chosen so that $\varepsilon^{1 / p}=r^{1 / q} c_{p}$, then

$$
\begin{gathered}
|f(t)|^{p} \leqq \varepsilon \int_{0}^{r}\left|f^{\prime}(t+s)\right|^{p} d s+K \int_{0}^{r}|f(t+s)|^{p} d s ; \\
\int_{N}^{\infty}|b(t) f(t)|^{p} d t \\
\leqq \int_{N}^{\infty} \int_{t}^{t+r}|b(t)|^{p}\left\{\varepsilon\left|f^{\prime}(s)\right|^{p}+K|f(s)|^{p}\right\} d s d t \\
=\int_{N}^{\infty} \int_{\max (s-r, N)}^{s}|b(t)|^{p}\left\{\varepsilon\left|f^{\prime}(s)\right|^{p}+K|f(s)|^{p}\right\} d t d s \\
\leqq\left\{\sup _{N \leqq s<\infty} \int_{s}^{s+r}|b(t)|^{p} d t\right\}\left\{\varepsilon \int_{N}^{\infty}\left|f^{\prime}(s)\right|^{p} d s+K \int_{N}^{\infty}|f(s)|^{p} d s\right\} .
\end{gathered}
$$

Lemma 5. Given $\varepsilon>0$, there exists $K(\varepsilon)>0$ such that

$$
\left\|D^{k} f\right\| \leqq \varepsilon\left\|D^{n} f\right\|+K(\varepsilon)\|f\|, f \in \mathscr{D}\left(D^{n}\right), 0 \leqq k<n,
$$

where the norms are taken in $L^{p}[0, \infty)$.

Proof. Let $[0, r]$ be a finite interval. Replacing $f$ by $f^{\prime}$ and proceeding as in the proof of Lemma 4, we arrive at the inequality

$$
\left|f^{\prime}(t)\right| \leqq C_{p} r^{1 / q}\left\{\int_{0}^{r}\left|f^{\prime \prime}(t+s)\right|^{p} d s+K_{0}(r) \int_{0}^{r}\left|f^{\prime}(t+s)\right|^{p} d s\right\}^{1 / p} .
$$

Suppose $\left\{f_{n}\right\}$ is a $D^{2}$-bounded sequence in $L^{p}[0, r]$. It is easy to see that the derivatives $f_{n}^{\prime}$ are uniformly bounded and equicontinuous on the interval $[0, r]$. Hence the operator $D$ in $L^{p}[0, r]$ is compact with respect to the operator $D^{2}$ in $L^{p}[0, r]$.

By Lemma 3(d), there exists a $K_{1}(r)>0$ such that

$$
K_{0}(r) \int_{0}^{r}\left|f^{\prime}(t+s)\right|^{p} d s \leqq \int_{0}^{r}\left|f^{\prime \prime}(t+s)\right|^{p} d s+K_{1}(r) \int_{0}^{r}|f(t+s)|^{p} d s .
$$

If $r$ is chosen so that $0<r<1$ and $\varepsilon^{1 / p}=2 C_{p} r^{1 / q}$, then the above inequalities yield the pointwise estimate 


$$
\left|f^{\prime}(t)\right|^{p} \leqq \varepsilon \int_{0}^{r}\left|f^{\prime \prime}(t+s)\right|^{p} d s+K(\varepsilon) \int_{0}^{r}|f(t+s)|^{p} d s .
$$

Integrating from 0 to $\infty$ and exchanging the order of integration, we arrive at the following inequality

$$
\|D f\|^{p} \leqq \varepsilon\left\|D^{2} f\right\|^{p}+K(\varepsilon)\|f\|^{p} .
$$

This is equivalent to an inequality of the form

$$
\|D f\| \leqq \varepsilon\left\|D^{2} f\right\|+K(\varepsilon)\|f\| \text {. }
$$

Inequalities of the form

$$
\left\|D^{k} f\right\| \leqq \varepsilon\left\|D^{k+1} f\right\|+K(\varepsilon)\|f\|
$$

follow easily by induction on $k$. Since $D^{k+1}$ is $D^{n}$-bounded, we finally obtain an inequality of the desired form

$$
\left\|D^{k} f\right\| \leqq \varepsilon\left\|D^{n} f\right\|+K(\varepsilon)\|f\| \text {. }
$$

Let $b$ be a measurable function on the interval $[0, \infty)$, and define the linear operator $B$ in $L^{p}[0, \infty)$ by

$$
\begin{aligned}
\mathscr{D}(B) & =\left\{f \in L^{p}[0, \infty): b f \in L^{p}[0, \infty)\right\}, \\
B f & =b \cdot f, f \in \mathscr{D}(B) .
\end{aligned}
$$

$B$ is closed and densely-defined.

In the following, $L_{\text {loc }}^{p}[\alpha, \infty)$ will denote the space of measurable functions on $[\alpha, \infty)$ which are locally in $L^{p}[\alpha, \infty)$.

Lemma 6. $B$ is $D$-defined if and only if $b \in L_{\mathrm{loc}}^{p}[0, \infty)$ and

$$
\lim _{s \rightarrow \infty} \sup \int_{s}^{s+1}|b(t)|^{p} d t<\infty \text {. }
$$

If $B$ is $D$-defined, then for every $\varepsilon>0$, there exists a $K(\varepsilon)>0$ such that

$$
\|B f\| \leqq \varepsilon\|D f\|+K(\varepsilon)\|f\|, f \in \mathscr{D}(D) .
$$

In particular, $D+B$ is closed on $\mathscr{D}(D)$.

Proof. Suppose that $B$ is $D$-defined. Since $B$ is closed, $B$ is $D$ bounded. Let $f$ be a $C^{\infty}$-function on $(-\infty, \infty)$ such that

$$
\begin{aligned}
0 & \leqq f \leqq 1 \\
f(s) & =1,0 \leqq s \leqq 1 \\
f(s) & =0,-\infty<s \leqq-1,2 \leqq s<\infty .
\end{aligned}
$$


Let $f_{s}(t)=f(t-s)$, and let $g_{s}$ be the restriction of $f_{s}$ to the interval $[0, \infty)$.

If $s \geqq 0$, then

$$
\begin{aligned}
\int_{s}^{s+1}\left|b(t) g_{s}(t)\right|^{p} d t & =\int_{s}^{s+1}|b(t)|^{p} d t \leqq\left\|b g_{s}\right\|^{p} \leqq\|B\|_{D}^{p}\left\|g_{s}\right\|^{p} \\
& \leqq\|B\|_{D}^{p} K_{p}\left\{\|f\|_{L^{p}(-\infty, \infty)}^{p}+\left\|f^{\prime}\right\|_{L^{p}(-\infty, \infty)}^{p}\right\},
\end{aligned}
$$

Hence $b \in L_{\mathrm{loc}}^{p}[0, \infty)$, and

$$
\lim _{s \rightarrow \infty} \sup \int_{s}^{s+1}|b(t)|^{p} d t<\infty .
$$

Conversely, suppose $b \in L_{\mathrm{loc}}^{p}[0, \infty)$ and

$$
\lim _{s \rightarrow \infty} \sup \int_{s}^{s+1}|b(t)|^{p} d t<\infty .
$$

Then

$$
\sup _{0 \leqq s<\infty} \int_{s}^{s+1}|b(t)|^{p} d t<\infty .
$$

It follows from Lemma 4 , with $N=0$, that $B$ is $D$-bounded and

$$
\|B f\| \leqq \varepsilon\|D f\|+K(\varepsilon)\|f\|, f \in \mathscr{D}(D) .
$$

By Lemma $3(\mathrm{a}), \quad D+B$ is closed.

LemMA 7. $B$ is $D$-compact if and only if $b \in L_{\mathrm{loc}}^{p}[0, \infty)$ and

$$
\lim _{s \rightarrow \infty} \int_{s}^{s+1}|b(t)|^{p} d t=0 \text {. }
$$

Proof. Suppose that $B$ is $D$-compact. By Lemma $6, b \in L_{\mathrm{loc}}^{p}[0, \infty)$. Suppose that there exists a sequence $s_{n} \rightarrow \infty$ and a $K>0$ such that

$$
\int_{s_{n}}^{s_{n}+1}|b(t)|^{p} d t \geqq K, \quad n=1,2, \cdots
$$

Let $\left\{g_{s_{n}}\right\}$ be the functions defined in the proof of Lemma 6; since $\left\{g_{s_{n}}\right\}$ is a $D$-bounded sequence, and $B$ is $D$-compact, we can assume that

$$
\left\|B g_{s_{n}}\right\| \underset{n \rightarrow \infty}{\longrightarrow} 0
$$

passing to a subsequence if necessary. On the other hand,

$$
\left\|B g_{s_{n}}\right\|^{p} \geqq \int_{s_{n}}^{s_{n}+1}|b(t)|^{p} d t \geqq K,
$$

a contradiction. Hence $\lim _{s \rightarrow \infty} \int_{s}^{s+1}|b(t)|^{p} d t=0$. 
Conversely, suppose that $b \in L_{\mathrm{oc}}^{p}[0, \infty)$ and that

$$
\lim _{s \rightarrow \infty} \int_{s}^{s+1}|b(t)|^{p} d t=0 \text {. }
$$

Let $\chi_{N}$ denote the characteristic function of $[0, N]$, and define

$$
B_{N} f=\chi_{N} b f, f \in \mathscr{D}(D) .
$$

By Lemma 4 , there is a constant $K>0$ such that

$$
\begin{aligned}
\left\|\left(B-B_{N}\right) f\right\|^{p} & =\int_{N}^{\infty}|b(t) f(t)|^{p} d t \\
& \leqq K\left\{\sup _{N \leqq s<\infty} \int_{s}^{s+1}|b(t)|^{p} d t\right\}\left\{\|D f\|^{p}+\|f\|^{p}\right\} .
\end{aligned}
$$

Hence $\left\|B-B_{N}\right\|_{D} \rightarrow 0$ as $N \rightarrow \infty$, so it suffices to show that each $B_{N}$ is $D$-compact.

For this purpose, let $\left\{f_{k}\right\}$ be a $D$-bounded sequence in $\mathscr{D}(D)$. Since

$$
\left|f_{k}(s)-f_{k}(t)\right|=\left|\int_{s}^{t} f_{k}^{\prime}(r) d r\right| \leqq|t-s|^{1 / q}\left\{\int_{s}^{t}\left|f^{\prime}(r)\right|^{p} d r\right\}^{1 / p},
$$

the $f_{k}$ are equicontinuous on $[0, N]$. If $\left\{f_{k,}\right\}_{j=1}^{\infty}$ is a subsequence which converges uniformly on $[0, N]$ then $\left\{B_{N} f_{k}\right\}_{j=1}^{\infty}$ converges in $L^{p}[0, \infty)$. Hence $B_{N}$ is $D$-compact.

THEOREM 3. Let $M$ be the maximal operator in $L^{p}[0, \infty), 1<p<\infty$, corresponding to the differential expression

$$
(m f)(t)=\sum_{j=0}^{n} a_{j} f^{(j)}(t), a_{j} \text { constants, } a_{n} \neq 0 .
$$

Let $B$ be the maximal operator in $L^{p}[0, \infty)$ corresponding to the differential expression

$$
\sum_{j=0}^{n-1} b_{j}(t) f^{(j)}(t)
$$

where the $b_{j}$ are measurable.

(a) $B$ is $M$-bounded if and only if $b_{j} \in L_{\mathrm{loc}}^{p}[0, \infty)$ and

$$
\lim _{s \rightarrow \infty} \sup \int_{s}^{s+1}\left|b_{j}(t)\right|^{p} d t<\infty, 0 \leqq j \leqq n-1 .
$$

(b) $B$ is $M$-compact if and only if $b_{j} \in L_{\mathrm{loc}}^{p}[0, \infty)$ and

$$
\lim _{s \rightarrow \infty} \int_{s}^{s+1}\left|b_{j}(t)\right|^{p} d t=0,0 \leqq j \leqq n-1 .
$$


(c) If $B$ is $M$-bounded, then for every $\varepsilon>0$ there exists $K(\varepsilon)>0$ such that

$$
\|B f\| \leqq \varepsilon\|M f\|+K(\varepsilon)\|f\|, f \in \mathscr{D}(M) .
$$

In particular, $M+B$ is closed on $\mathscr{D}(M)$.

Proof. Suppose that $B$ is $M$-bounded. If the functions $g_{s}$ are constructed as in the proof of Lemma 6 , we have

$$
\begin{aligned}
& \sup _{s \geq 0} \int_{s}^{s+1}\left|b_{0}(t)\right|^{p} d t=\sup \int_{s}^{s+1}\left|B g_{s}(t)\right|^{p} d t \\
& \quad \leqq \sup _{s \geqq 0}\left\|B g_{s}\right\|^{p} \leqq \sup _{s \geqq 0}\|B\|_{M}^{p}\left(\left\|M g_{s}\right\|+\left\|g_{s}\right\|\right)^{p}<\infty .
\end{aligned}
$$

Hence $b_{0} \in L_{\mathrm{loc}}^{p}[0, \infty)$ and

$$
\limsup _{s \rightarrow \infty} \int_{s}^{s+1}\left|b_{0}(t)\right|^{p} d t<\infty .
$$

Let $1 \leqq k \leqq n-1$ and assume that $b_{j} \in L_{\mathrm{loc}}^{p}[0, \infty)$ and

$$
\limsup _{s \rightarrow \infty} \int_{s}^{s+1}\left|b_{j}(t)\right|^{p} d t<\infty, 0 \leqq j \leqq k-1 \text {. }
$$

The functions $g_{s}$ can be altered so that

$$
g_{s}^{(k)}(t)=1, s \leqq t \leqq s+1 .
$$

The same type of estimate as used in the preceding paragraph yields the results

$$
b_{k} \in L_{\mathrm{loc}}^{p}[0, \infty)
$$

and

$$
\limsup _{s \rightarrow \infty} \int_{s}^{s+1}\left|b_{k}(t)\right|^{p} d t<\infty .
$$

By induction, this holds for all $k, 0 \leqq k \leqq n-1$.

Conversely, assume $b_{j}(t) \in L_{\mathrm{loc}}^{p}[0, \infty)$ and

$$
\limsup _{s \rightarrow \infty} \int_{s}^{s+1}\left|b_{j}(t)\right|^{p} d t<\infty, 0 \leqq j \leqq n-1 .
$$

Let $B_{j}$ be the maximal operator corresponding to the expression $b_{j}(t) f^{(j)}(t)$.

By Lemma 6,

$$
\left\|B_{j} f\right\| \leqq \varepsilon_{j}\left\|D^{j+1} f\right\|+K_{0}\left(\varepsilon_{j}\right)\left\|D^{j} f\right\|, f \in \mathscr{D}\left(D^{j+1}\right) .
$$


From Lemma 5 we can deduce an inequality of the form

$$
\left\|B_{j} f\right\| \leqq \varepsilon_{j}\left\|D^{n} f\right\|+K\left(\varepsilon_{j}\right)\|f\|, f \in \mathscr{D}\left(D^{n}\right) .
$$

Summing over $j$ we arrive at an inequality of the form

$$
\|B f\| \leqq \varepsilon\left\|D^{n} f\right\|+K(\varepsilon)\|f\|, f \in \mathscr{D}\left(D^{n}\right) .
$$

Since $M$ is a polynomial in $D$ of order $n, \mathscr{D}(M)=\mathscr{D}\left(D^{n}\right)$, and the $M$-topology is equivalent with the $D^{n}$-topology for $\mathscr{D}(M)$. Hence we get an inequality of the desired form,

$$
\|B f\| \leqq \varepsilon\|M f\|+K(\varepsilon)\|f\| \text {. }
$$

By Lemma $3(\mathrm{a}), M+B$ is closed on $\mathscr{D}(M)$. This completes the proof of parts (a) and (c) of the theorem.

If

$$
\lim _{s \rightarrow \infty} \int_{s}^{s+1}\left|b_{j}(t)\right|^{p} d t=0,0 \leqq j \leqq n-1,
$$

then each $B_{j}$ is $D^{j+1}$-compact, by Lemma 7. And so $B_{j}$ is $D^{n}$-compact, therefore $M$-compact. Hence $B$ is $M$-compact.

Conversely, if $B$ is $M$-compact, then the relations

$$
\lim _{s \rightarrow \infty} \int_{s}^{s+1}\left|b_{k}(t)\right|^{p} d t=0
$$

can be proved by induction on $k$ as in the proof of part (a) and of Lemma 7.

THEOREM 4. Let $M$ and $L$ be the maximal operators in $L^{p}[0, \infty)$, $1<p<\infty$, corresponding to the differential expressions

$$
\begin{aligned}
(m f)(t) & =\sum_{j=0}^{n} a_{j} f^{(j)}(t), \quad a_{j} \text { constants, } a_{n} \neq 0, \\
(l f)(t) & =(m f)(t)+\sum_{j=0}^{n} b_{j}(t) f^{(j)}(t) .
\end{aligned}
$$

Suppose $b_{n}$ is continuous and satisfies

$$
\begin{gathered}
b_{n}(t) \neq-a_{n}, 0 \leqq t<\infty \\
\lim _{t \rightarrow \infty} b_{n}(t)=0 .
\end{gathered}
$$

Suppose $b_{j} \in L_{\mathrm{loc}}^{p}[0, \infty)$ and satisfies

$$
\lim _{s \rightarrow \infty} \int_{s}^{s+1}\left|b_{j}(t)\right|^{p} d t=0, \quad 0 \leqq j \leqq n-1 .
$$

Then $\mathscr{D}(L)=\mathscr{D}(M)$, and $\sigma_{e}(l)=\sigma_{e}(m)$. If $\lambda \in \rho_{e}(m), \kappa(\lambda I-M)=$ $\kappa(\lambda I-L)$. 
Proof. Let $B_{n}$ be the maximal operator corresponding to the expression $b_{n}(t) f^{(n)}(t) .^{2}$ In view of Theorem 3 and Lemma 3 it suffices to prove the theorem in the case

$$
(l f)(t)=(m f)(t)+b_{n}(t) f^{(n)}(t) .
$$

So we assume $b_{j}(t)=0,0 \leqq j \leqq n-1$. Since the essential spectrum and the Fredholm index are localizable to the endpoint $\infty$, and since the graph topologies of $\mathscr{D}(L)$ and $\mathscr{D}(M)$ are equivalent on compact subsets of $[0, \infty)$, we may assume, by passing to an interval of the form $[N, \infty)$, that $\left|b_{n}(t)\right| \leqq \varepsilon, 0 \leqq t<\infty$.

We have

$$
\begin{aligned}
\left\|B_{n} f\right\| & =\left\{\int_{0}^{\infty}\left|b_{n}(t) f^{(n)}(t)\right|^{p} d t\right\}^{1 / p} \\
& \leqq \varepsilon\left\|D^{n} f\right\| \leqq \varepsilon\left\|D^{n}\right\|_{\mathcal{M}}(\|M f\|+\|f\|) .
\end{aligned}
$$

If $\varepsilon$ is sufficiently small, Lemma $3($ a) applies, and $\mathscr{D}(L)=\mathscr{D}(M)$. Also, by Lemma 3(c) and suitable choice of $\varepsilon$, we must have $\sigma_{e}(l)=$ $\sigma_{e}(m)$.

Now suppose $\left|b_{n}(t)\right|<\left|a_{n}\right|, 0 \leqq t<\infty$, so that the hypotheses of the theorem are satisfied for

$$
\left(l_{\beta} f\right)(t)=(m f)(t)+\beta b_{n}(t) f^{(n)}(t),
$$

where $0 \leqq \beta \leqq 1$. We have shown that $\sigma_{e}\left(l_{\beta}\right)=\sigma_{e}(m)$, so that the function $\beta \rightarrow \kappa\left(\lambda I-L_{\beta}\right)$ is well-defined, $\lambda \in \rho_{e}(m)$. This function is continuous and integervalued, hence a constant. In particular, $\kappa(\lambda I-M)=\kappa(\lambda I-L)$.

\section{Perturbation of the Euler operator.}

THEOREM 5. Let $L$ be the maximal operator in $L^{p}[1, \infty), 1<p<\infty$, corresponding to the Euler differential expression

$$
(l f)(t)=\sum_{j=0}^{n} b_{j} t^{j} f^{(j)}(t), \quad b_{j} \text { constants, } b_{n} \neq 0 .
$$

Let $C$ be the maximal operator in $L^{p}[1, \infty)$ corresponding to the expression

$$
\sum_{j=0}^{n-1} c_{j}(t) t^{j} f^{(j)}(t)
$$

where the $c_{j}$ are measurable.

(a) $C$ is $L$-bounded if and only if $c_{j} \in L_{\mathrm{loc}}^{p}[1, \infty)$ and

$$
\limsup _{s \rightarrow \infty} \int_{s}^{\alpha s} \frac{1}{t}\left|c_{j}(t)\right|^{p} d t<\infty \text { for some } \alpha>1,0 \leqq j \leqq n-1
$$

2 Professor S. Goldberg pointed out, that the proof was incomplete. The remaining part can be found at the end of the paper. 
(b) $C$ is $L$-compact if and only if $c_{j} \in L_{\mathrm{loc}}^{p}[1, \infty)$ and $\lim _{s \rightarrow \infty} \int_{s}^{a s} \frac{1}{t}\left|c_{j}(t)\right|^{p} d t=0$ for some $\alpha>1,0 \leqq j<n-1$.

(c) If $C$ is L-bounded, then for every $\varepsilon>0$ there exists $K(\varepsilon)>0$ such that

$$
\|C f\| \leqq \varepsilon\|L f\|+K(\varepsilon)\|f\|, f \in \mathscr{D}(L) .
$$

In particular, $L+C$ is closed on $\mathscr{D}(L)$.

Proof. Let $M$ be the maximal operator in $L^{p}[0, \infty)$ corresponding to the differential expression

$$
(m f)(t)=b_{0}+\sum_{j=1}^{n} b_{j} \prod_{k=0}^{j-1}\left(\frac{d}{d s}-\left(\frac{1}{p}+k\right)\right),
$$

and let $B$ be the maximal operator in $L^{p}[0, \infty)$ corresponding to the expression

$$
c_{0}\left(e^{s}\right)+\sum_{j=1}^{n-1} c_{j}\left(e^{s}\right) \prod_{h=0}^{j-1}\left(\frac{d}{d s}-\left(\frac{1}{p}+k\right)\right) .
$$

Let $\tau$ be the isometry of $L^{p}[1, \infty)$ and $L^{p}[0, \infty)$ introduced in the proof of Theorem 2. Then

$$
L=\tau^{-1} M \tau
$$

and

$$
C=\tau^{-1} B \tau
$$

Also,

$$
\int_{s}^{\alpha s} \frac{1}{t}\left|c_{j}(t)\right|^{p} d t=\int_{e^{s}}^{e^{s}+e^{\alpha}}\left|c_{j}(u)\right|^{p} d u
$$

Combining Theorem 3 and a downward induction argument on the coefficients $c_{j}$, we arrive at parts (a) and (b) of Theorem 5. Part (c). also follows from Theorem 3 .

Theorem 6. Let $L$ and $M$ be the maximal operators in $L^{p}[1, \infty)$, $1<p<\infty$, corresponding to the differential expressions

$$
\begin{aligned}
& (l f)(t)=\sum_{j=0}^{n} b_{j} t^{j} f^{(j)}(t), b_{j} \text { constants, } b_{n} \neq 0 . \\
& (m f)(t)=(l f)(t)+\sum_{j=0}^{n} c_{j}(t) t^{j} f^{(j)}(t) .
\end{aligned}
$$


Suppose $c_{n}$ is continuous and satisfies

$$
\begin{gathered}
c_{n}(t) \neq-b_{n}, 1 \leqq t<\infty \\
\lim _{t \rightarrow \infty} c_{n}(t)=0 .
\end{gathered}
$$

Suppose $c_{j} \in L_{\mathrm{loc}}^{p}[0, \infty)$ and satisfies

$$
\lim _{s \rightarrow \infty} \int_{s}^{\alpha s} \frac{1}{t}\left|c_{j}(t)\right|^{p} d t=0 \text { for some } \alpha>1,0 \leqq j \leqq n-1 .
$$

Then $\mathscr{D}(L)=\mathscr{D}(M)$, and $\sigma_{e}(l)=\sigma_{e}(m) . \quad$ If $\lambda \in \rho_{e}(m), \kappa(\lambda I-M)=$ $\kappa(\lambda I-L)$.

Proof. A straightforward verification, as in the proof of Theorem 5 , shows that the transform of Theorem 6 under $\tau$ is Theorem 4 .

7. Some special cases. The perturbation criterion of Theorem 5 includes all functions $c(t)$ such that $t^{-1 / p} c(t) \in L^{p}[1, \infty)$. It includes all bounded measurable functions with limit zero at $\infty$. The criterion shows, for instance, that if $\alpha<j<n$, then $t^{\alpha} f^{(j)}$ is compact with respect to the Euler operator of degree $n$. If $\alpha<n$, Theorem 6 shows, that $t^{\alpha} f^{(n)}$ has no effect on the essential spectrum of $l$. In particular, if

$$
(m f)(t)=\sum_{j=0}^{n} a_{j}(t) f^{(j)}(t)
$$

is a Fuchsian differential expression, where $a_{n}(t)=0\left(t^{n}\right)$, then $m$ can be written in the form of Theorem 6 , and the essential spectrum of $m$ can be determined from the coefficients as in Theorem 2 .

For instance, consider the Riemann differential expression

$$
(m f)(t)=t(t+1) f^{\prime \prime}(t)+(a t+b) f^{\prime}(t)+\frac{c t^{2}+d t+e}{t(t+1)} f(t) .
$$

Except for the change of variable $t \rightarrow-t$ this is the equation investigated by Rota [10]. By Theorem $6, \sigma_{e}(m)=\sigma_{e}(l)$, where

$$
(l f)(t)=t^{2} f^{\prime \prime}(t)+a t f^{\prime}(t)+c f(t) .
$$

By Theorem 2,

$$
\sigma_{e}(l)=\{d(i r):-\infty<r<\infty\},
$$

where

$$
d(z)=\left(z-\frac{1}{p}\right)\left(z-\frac{1}{p}-1\right)+a\left(z-\frac{1}{p}\right)+c .
$$


Hence

$\sigma_{e}(m)=\left\{-r^{2}+i r\left(\alpha-1-\frac{2}{p}\right)+\frac{1}{p^{2}}+(1-\alpha) \frac{1}{p}+c:-\infty<r<\infty\right\}$.

This is equivalent to the expression obtained by Rota.

8. Remarks.

(a) The Euler operator in $L^{p}(0,1]$.

The mapping $\tau$ defined as in the proof of Theorem 2 by

$$
\tau f(s)=e^{s / p} f\left(e^{s}\right)
$$

also establishes an isometric isomorphism of $L^{p}(0,1]$ and $L^{p}(-\infty, 0]$.

The Euler operator

$$
L=\sum_{j=0}^{n} a_{j} t^{j} D^{j}
$$

in $L^{p}(0,1]$ is isometric isomorphic via $\tau$ to the constant coefficient operator

$$
M=a_{0}+\sum_{j=1}^{n} a_{j} \prod_{k=0}^{j-1}\left(D-\left(\frac{1}{p}+k\right)\right)
$$

in $L^{p}(-\infty, 0]$.

The operator $D$ in $L^{p}(-\infty, 0]$ is isometric isomorphic to the operator $(-D)$ in $L^{p}[0, \infty)$; therefore $D$ in $L^{p}(-\infty, 0]$ has the essential spectrum $\{i t:-\infty<t<\infty\}$, and the Fredholm index of $\lambda I-D$ is 0 for $\mathscr{R} e \lambda<0$ and 1 for $\mathscr{R} e \lambda>0$.

It follows, that $l$ on the interval $(0,1]$ has the same essential spectrum as $l$ on the interval $[1, \infty)$ and the Fredholm index of $\lambda I-L$ is the number of roots of the polynomial $d(z)-\lambda$ of Theorem 2 , counted with multiplicity, which lie in the half-plane $\mathscr{R}_{e} z>0$.

The perturbation results also carry over to the interval $(0,1]$. The Theorems of $\S 6$ are true for the operator $L$ in $L^{p}(0,1]$, when 1 is substituted for 0 and 0 for $\infty$, in particular we now take the limes and $\lim \sup$ of $\int_{s}^{\alpha s}(1 / u)|c(u)|^{p} d u$ as $s \rightarrow 0$.

The Euler operator $L$ in $L^{p}(0, \infty)$ is isometric isomorphic via $\tau$ to the constant coefficient operator considered above in $L^{p}(-\infty, \infty)$, and the essential spectrum is given by the same formula. The Fredholm index is $0, \sigma_{e}(L)=\sigma(L)$ and $L_{0}=L$.

(b) The condition

$$
\limsup _{s \rightarrow \infty} \int_{s}^{\alpha s} \frac{1}{t}|b(t)|^{p} d t<\infty \text { for some } \alpha>1
$$


is equivalent to the condition

$$
\limsup _{s \rightarrow \infty} s \int_{s}^{\infty} \frac{1}{t^{2}}|b(t)|^{p} d t<\infty
$$

Also, the condition

$$
\lim _{s \rightarrow \infty} \int_{s}^{\alpha s} \frac{1}{t}|b(t)|^{p} d t=0 \text { for some } \alpha>1
$$

is equivalent to the condition

$$
\lim _{s \rightarrow \infty} s \int_{s}^{\infty} \frac{1}{t^{2}}|b(t)|^{p} d t=0
$$

This second set of conditions could just as well have been used in Theorem 5 and 6.

The proof of these assertions follows from the inequalities

$$
\begin{gathered}
\sup _{s \geqq N} \int_{s}^{\alpha s} \frac{1}{t}|b(t)|^{p} d t \leqq \sup _{s \geqq N} \alpha s \int_{s}^{\alpha s} \frac{1}{t^{2}}|b(t)|^{p} d t \\
\leqq \alpha \sup _{s \geqq N} s \int_{s}^{\infty} \frac{1}{t^{2}}|b(t)|^{p} d t,
\end{gathered}
$$

and

$$
\begin{gathered}
\sup _{s \geqq N} s \int_{s}^{\infty} \frac{1}{t^{2}}|b(t)|^{p} d t=\sup _{s \geqq N} s \sum_{n=0}^{\infty} \int_{\alpha^{n} s_{s}}^{\alpha^{n+1} s} \frac{1}{t^{2}}|b(t)|^{p} d t \\
\quad \leqq \sup _{s \geqq N} s \sum_{n=0}^{\infty} \frac{1}{\alpha^{n} s} \int_{\alpha^{n} n_{s}}^{\alpha^{n+1} s} \frac{1}{t}|b(t)|^{p} d t \\
\quad \leqq \frac{\alpha}{\alpha-1} \sup _{s \geqq N} \int_{s}^{\alpha s} \frac{1}{t}|b(t)|^{p} d t .
\end{gathered}
$$

(c) A basis of solutions $f_{1}(\lambda, t), \cdots, f_{n}(\lambda, t)$ of a differential equation $l(f)=\lambda f$ of order $n$ is said to be a norm-analytic basis at $\lambda_{0}$ if there is a neighborhood $N$ of $\lambda_{0}$ such that (i) the functions $f_{i}$ are analytic in $\lambda$ for $\lambda \in N$ and (ii) there is an integer $k$ such that for each $\lambda \in N,\left\{f_{i}\right\}_{i=1}^{k}$ span the set of solutions of $l(f)=\lambda f$ which lie in $L^{p}$. In [10], Rota proved the following criterion:

LEMma. If at $\lambda$ either the differential operator $l$ in $L^{p}$ or its adjoint $l^{*}$ in $L^{q},(1 / p)+(1 / q)=1$ (cf [9], for definition of adjoint), does not have a norm-analytic basis of solutions, then $\lambda$ belongs to the essential spectrum of $l$.

If $l$ is the Euler differential expression of Theorem 2, the equation $l(y)=\lambda y$ has solutions $\varphi_{j}(t)=t^{\alpha_{j}}$, where $\alpha_{j}$ is a root of the algebraic 
equation

$$
b_{n} z(z-1) \cdots(z-n+1)+\cdots+b_{1} z+b_{0}=\lambda .
$$

Now $\varphi_{j} \in L^{p}[1, \infty)$ if and only if $\mathscr{R}_{e}\left(\alpha_{j}\right)<-(1 / p)$.

Hence $l$ will not have a norm-analytic basis at any point of the curve

$$
\begin{aligned}
\lambda=b_{n}\left(i r-\frac{1}{p}\right)\left(i r-\frac{1}{p}-1\right) \cdots\left(i r-\frac{1}{p}-n+1\right) & \\
& +\cdots+b_{1}\left(i r-\frac{1}{p}\right)+b_{0}, \quad-\infty<r<\infty .
\end{aligned}
$$

This curve is identical to $\{d(i r):-\infty<r<\infty\}$, where $d(z)$ is the polynomial defined in Theorem 2.

If $\lambda$ is not on this curve, then it can be shown that the resolvent. operator $(\lambda I-l)^{-1}$ is a sum of integral operators whose kernels are of the Hardy-Littlewood-Polya type (cf [7], or [5] pp. 531-532). This yields another proof of Theorem 2 , but the details are more complicated.

This method also shows that the essential spectrum of the Euler operator is precisely the set of points at which $l$ or $l^{*}$ does not have a norm-analytic basis of solutions. That this is not true in general is shown by the following example.

Define

$$
(l f)(t)=f^{\prime}(t)+(\sin t+t \cos t) f(t), 0 \leqq t<\infty .
$$

The equation $l f=\lambda f$ has the solution

$$
\varphi_{\lambda}(t)=\exp [t(\lambda-\sin t)],
$$

while the adjoint equation $l^{*} g=\lambda g$ has the solution

$$
\psi_{\lambda}(t)=1 / \varphi_{\lambda}(t) \text {. }
$$

Now $\varphi_{\lambda} \in L^{p}[0, \infty)$ if $\mathscr{R e}(\lambda)<-1$ and $\varphi_{\lambda} \notin L^{p}[0, \infty)$ if $\mathscr{R e}(\lambda)>-1$, so $l$ does not have a norm-analytic basis on the line $\mathscr{R}(\lambda)=-1$.

Similarly, $l^{*}$ does not have a norm-analytic basis on the line $\mathscr{R}(\lambda)=1 . \quad l$ and $l^{*}$ have norm-analytic bases if $\mathscr{R e}(\lambda) \neq \pm 1$.

Since 0 is a regular enojpoint for the differential expression $l$, a necessary condition that a point $\lambda$ be in $\rho_{e}(l)$ is that either $\varphi_{\lambda} \in L^{p}[0, \infty)$ or $\psi_{\lambda} \in L^{q}[0, \infty),(1 / p)+(1 / q)=1$ (cf [9]). Hence the entire strip $\left\{-1 \leqq \mathscr{R}_{e}(\lambda) \leqq 1\right\}$ is contained in the essential spectrum of $l$. It is easy to see that $\sigma_{e}(l)$ actually coincides with this vertical strip.

It seems possible that the boundary of the essential spectrum of an arbitrary differential expression consists of points $\lambda$ at which either $l f=\lambda f$ or $l^{*} g=\lambda g$ does not have a norm-analytic basis of solutions. 
(d) The fact that the isomorphism $(\tau f)(s)=e^{s / p} f\left(e^{s}\right)$ converts a resolvent operator of Hardy-Littlewood-Polya type into a resolvent operator of convolution type is a special case of the following situation.

Let $K$ be a measurable function on $[0, \infty)$, and let

$$
(T f)(x)=\frac{1}{x} \int_{0}^{x} K\left(\frac{y}{x}\right) f(y) d y, f \in L^{p}[0, \infty) .
$$

The mapping $\tau$ may be regarded as an isometric isomorphism of $L^{p}[0, \infty)$ and $L^{p}(-\infty, \infty)$.

The operator $S=\tau T \tau^{-1}$ in $L^{p}(-\infty, \infty)$ is given by

$$
(S g)(\omega)=\int_{-\infty}^{\infty} K\left(e^{z-\omega}\right) e^{((1 / p)-1)(\omega-z)} g(z) d z .
$$

$S$ is a convolution operator with kernel

$$
J(r)=K\left(e^{-r}\right) e^{((1 / p)-1) r} .
$$

Conversely, a convolution operator in $L^{p}(-\infty, \infty)$ with kernel $J$ determines a Hardy-Littlewood-Polya operator in $L^{p}[0, \infty)$ with kernel

$$
K(s)=s^{(1 / p)-1} J(-\log s) .
$$

The norm of $S$ is at most the $L^{1}$-norm of $J$. Hence if

$$
\int_{-\infty}^{\infty}|J(r)| d r=\int_{0}^{\infty}|K(s)| s^{-1 / p} d s<\infty,
$$

then $T$ is bounded, and

$$
\|T\| \leqq \int_{0}^{\infty}|K(s)| s^{-1 / p} d s .
$$

This last statement is just the Hardy-Littlewood-Polya inequality (cf [7]).

Added in Proof. Professor S. Goldberg has pointed out that the proof that $D(L)=D(M)$ in Theorem 4 is incomplete, i.e., it must be shown that $f \in L^{p}$ and $l f \in L^{p}$ imply $m f \in L^{p}$. This follows easily with the aid of a more general form of theorem 3(c), namely, that inequalities of the form

$$
\|B f\| \leqq \epsilon\|m f\|+K\|f\|
$$

obtain, where the norm is taken in $L^{p}[0, N)$ for $1 \leqq N \leqq \infty$, and $K$ depends on $\epsilon$ and $p$ but not on $N$. These inequalities result from modifying and sharpening the proofs of $\S 5$. 


\section{BIBLIOGRAPHY}

1. F. Atkinson, Normal solvability of linear equations in normed spaces, Mat. Sb. 28 (1951), 3-14.

2. E. Balslev, Perturbation of ordinary differential operators, to appear, Math. Scand.

3. E. Balslev, Perturbation of differential operators, Ph. D. thesis (1963), University of California, Berkeley, Calif.

4. F. E. Browder, On the spectral theory of elliptic differential operators I, Math., Ann., 142 (1961), 22-130.

5. Dunford and Schwartz, Linear Operators, Part I, Interscience Press, 1958.

6. I. C. Gokhberg and M. G. Krein, Fundamental theorems on deficiency numbers, root numbers and indices of linear operators, Uspekhi Mat. Nauk, 12 (1957), 43-118, (Am. Math. Soc. Translations, Series 2, vol. 13).

7. Hardy, Littlewood and Polya, Inequalities, Cambridge, 1952.

8. T. Kato, Perturbation theory for nullity, deficiency and other quantities of linear operators, J. d'Analyse Math., 6 (1958), 261-322.

9. G. C. Rota, Extension theory of differential operators, Communs. Pure App. Math. 11 (1958), 23-65.

10. G. C. Rota, On the spectra of singular boundary value problems, J. Math. and Mech., 10 (1961), 83-90.

11. M. S. Birman, On the spectrum of singular boundary value problems, Mat. Sbornik T. 55 (2) 97 (1961), 125-174. 


\section{PACIFIC JOURNAL OF MATHEMATICS}

\section{EDITORS}

\author{
Robert Osserman \\ Stanford University \\ Stanford, California
M. G. Arsove
University of Washington
Seattle 5 , Washington

\author{
J. DugundJI \\ University of Southern California \\ Los Angeles 7, California
}

Lowell J. Paige

University of California

Los Angeles 24, California

\section{ASSOCIATE EDITORS}
E. F. BECKENBACH
B. H. NeumanN
F. WOLF
K. YOSIDA

\section{SUPPORTING INSTITUTIONS}

\author{
UNIVERSITY OF BRITISH COLUMBIA \\ CALIFORNIA INSTITUTE OF TECHNOLOGY \\ UNIVERSITY OF CALIFORNIA \\ MONTANA STATE UNIVERSITY \\ UNIVERSITY OF NEVADA \\ NEW MEXICO STATE UNIVERSITY \\ OREGON STATE UNIVERSITY \\ UNIVERSITY OF OREGON \\ OSAKA UNIVERSITY \\ UNIVERSITY OF SOUTHERN CALIFORNIA
}

\author{
STANFORD UNIVERSITY \\ UNIVERSITY OF TOKYO \\ UNIVERSITY OF UTAH \\ WASHINGTON STATE UNIVERSITY \\ UNIVERSITY OF WASHINGTON \\ * * * * \\ AMERICAN MATHEMATICAL SOCIETY \\ CALIFORNIA RESEARCH CORPORATION \\ SPACE TECHNOLOGY LABORATORIES \\ NAVAL ORDNANCE TEST STATION
}

Mathematical papers intended for publication in the Pacific Journal of Mathematics should by typewritten (double spaced), and on submission, must be accompanied by a separate author's résumé. Manuscripts may be sent to any one of the four editors. All other communications to the editors should be addressed to the managing editor, L. J. Paige at the University of California, Los Angeles 24, California.

50 reprints per author of each article are furnished free of charge; additional copies may be obtained at cost in multiples of 50 .

The Pacific Journal of Mathematics is published quarterly, in March, June, September, and December. Effective with Volume 13 the price per volume (4 numbers) is $\$ 18.00$; single issues, $\$ 5.00$. Special price for current issues to individual faculty members of supporting institutions and to individual members of the American Mathematical Society: $\$ 8.00$ per volume; single issues $\$ 2.50$. Back numbers are available.

Subscriptions, orders for back numbers, and changes of address should be sent to Pacific Journal of Mathematics, 103 Highland Boulevard, Berkeley 8, California.

Printed at Kokusai Bunken Insatsusha (International Academic Printing Co., Ltd.), No. 6, 2-chome, Fujimi-cho, Chiyoda-ku, Tokyo, Japan.

PUBLISHED BY PACIFIC JOURNAL OF MATHEMATICS, A NON-PROFIT CORPORATION

The Supporting Institutions listed above contribute to the cost of publication of this Journal, but they are not owners or publishers and have no responsibility for its content or policies. 


\section{Pacific Journal of Mathematics}

\section{Vol. 14, No. $3 \quad$ July, 1964}

Erik Balslev and Theodore William Gamelin, The essential spectrum of a class of ordinary differential operators . . . . . . . . . . . . . . . . . . . .

James Henry Bramble and Lawrence Edward Payne, Bounds for derivatives in

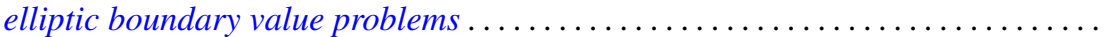

Hugh D. Brunk, Integral inequalities for functions with nondecreasing

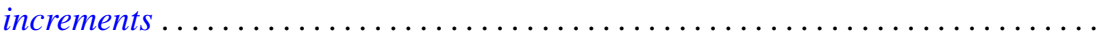

William Edward Christilles, A result concerning integral binary quadratic

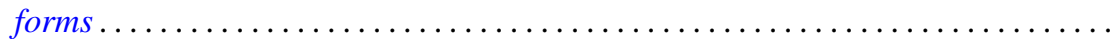

Peter Crawley and Bjarni Jónsson, Refinements for infinite direct decompositions of

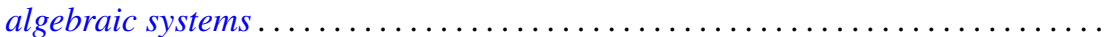

Don Deckard and Carl Mark Pearcy, On continuous matrix-valued functions on a Stonian space.

Raymond Frank Dickman, Leonard Rubin and P. M. Swingle, Another

characterization of the $n$-sphere and related results $\ldots \ldots \ldots \ldots \ldots \ldots$

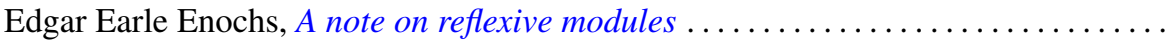

Vladimir Filippenko, On the reflection of harmonic functions and of solutions of the

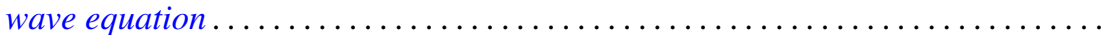

Derek Joseph Haggard Fuller, Mappings of bounded characteristic into arbitrary

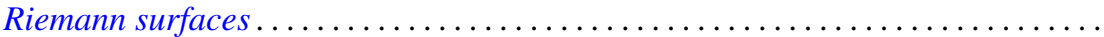
895

Curtis M. Fulton, Clifford vectors . . . . . . . . . . . . . . . . . . . . . . . . . . . . . . 917

Irving Leonard Glicksberg, Maximal algebras and a theorem of Radó . .

919

Kyong Taik Hahn, Minimum problems of Plateau type in the Bergman metric

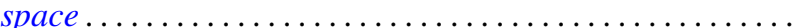

A. Hayes, A representation theory for a class of partially ordered rings...

J. M. C. Joshi, On a generalized Stieltjes trasform

J. M. C. Joshi, Inversion and representation theorems for a generalized Laplace transform ...

Eugene Kay McLachlan, Extremal elements of the convex cone $B_{n}$ of functions ...

Robert Alan Melter, Contributions to Boolean geometry of p-rings ...

James Ronald Retherford, Basic sequences and the Paley-Wiener criterion . . . . . . . 1019

Dallas W. Sasser, Quasi-positive operators. .

Oved Shisha, On the structure of infrapolynomials with prescribed coefficients ..

Oved Shisha and Gerald Thomas Cargo, On comparable means

Maurice Sion, A characterization of weak ${ }^{*}$ convergence ........

Morton Lincoln Slater and Robert James Thompson, A permanent inequality for

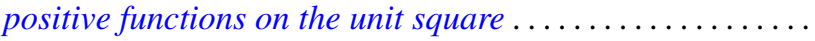

David A. Smith, On fixed points of automorphisms of classical Lie algebras ...

Sherman K. Stein, Homogeneous quasigroups ................

J. L. Walsh and Oved Shisha, On the location of the zeros of some infrapolynomials with prescribed coefficients .

Ronson Joseph Warne, Homomorphisms of $d$-simple inverse semigroups with identity . 